\title{
Position of the dentifera-group in the Coronatella- branch and its relocation to a new genus: Magnospina gen. n. (Crustacea, Chydoridae, Aloninae)
}

Francisco Diogo R. Sousa ${ }^{1,2}$, Lourdes Maria Abdu Elmoor-Loureiro², Sandro Santos ${ }^{1}$

I Núcleo de Estudos em Biodiversidade Aquática, Programa de Pós-graduação em Biodiversidade Animal, Universidade Federal de Santa Maria - UFSM, Av. Roraima 1000, Camobi, CEP 97105-900, Santa Maria, RS, Brazil 2 Laboratório de Biodiversidade Aquática, Universidade Católica de Brasilia - UCB, QS7 lote 1, Bloco M, sala 204, CEP 71966-700, Taguatinga, DF, Brazil

Corresponding author: Francisco Diogo R. Sousa (sousa_bio@yahoo.com.br; fdiogo.rs@gmail.com)

Academic editor: A. Myers | Received 22 February 2016 | Accepted 2 April 2016 | Published 4 May 2016

http://zoobank.org/D247100A-6343-4E19-BCDA-93B8E3122D10

Citation: Sousa FDR, Elmoor-Loureiro LMA, Santos S (2016) Position of the dentifera-group in the Coronatella-branch and its relocation to a new genus: Magnospina gen. n. (Crustacea, Chydoridae, Aloninae). ZooKeys 586: 95-119. doi: 10.3897/zookeys.586.8209

\begin{abstract}
Magnospina gen. n. was created to relocate species of the dentifera-group from Alona sensu lato (Crustacea: Cladocera) and include Magnospina dentifera comb. n. and Magnospina siamensis comb. $\mathbf{n}$. The synapomorphies of the Magnospina gen. n. are (1) basal spines longer than 2/3 of the postabdominal claw, (2) presence of 1-4 large denticles, broad at their bases, protruding downwards, without setules between them. Morphological traits such as habitus, rostrum and postabdomen shape, armature of IDL setae, number of setae on the exopod of limb III are also important in the distinction between Magnospina gen. $\mathbf{n}$. and other genera from the Coronatella-branch. The morphology of Magnospina dentifera comb. n. male confirms the closer relationship with the clade composed by the elgans-group from Alona sensu lato, Ovalona and Leberis, but the external morphology, morphology of the postabdominal claw, basal spine and setae 2-3 of IDL support their separation from any of the group cited. It is concluded that the Coronatella-lineage of Aloninae is composed of the genera Coronatella, Anthalona, Karualona, Bergamina, Extremalona, Ovalona, Celsinotum, Leberis and Magnospina gen. n. The elegans-group from Alona sensu lato also belongs to Coronatella-lineage, but still need formal allocation.
\end{abstract}

\section{Keywords}

Alona broaensis, Alona dentifera, Alona siamensis, Celsinotum, Leberis, male, morphology, Ovalona

Copyright Francisco Diogo R. Sousa et al. This is an open access article distributed under the terms of the Creative Commons Attribution License (CC BY 4.0), which permits unrestricted use, distribution, and reproduction in any medium, provided the original author and source are credited. 


\section{Introduction}

The taxonomic status of Alona dentifera (Sars, 1901) (Crustacea: Cladocera) was discussed by Sinev et al. (2004). In this study, the authors relocated Alonella dentifera to the genus Alona Baird, 1843 based on the absence of typical morphological traits of the subfamily Chydorinae Dybowsky \& Grochowski, 1894 emend. Frey, 1967 and presence of some morphological traits of Aloninae Dybowsky \& Grochowski, 1894 emend. Frey, 1967. Because of the polyphyletic nature of Alona (Sacherová and Hebert 2003, Elmoor-Loureiro 2004, Eliáz-Gutiérrez et al. 2008; Van Damme et al. 2010), allocations of species groups to different genera were made: Phreatalona, which corresponds to the protzi-complex (Van Damme et al. 2009); Coronatella, which corresponds to the rectangula-complex (Van Damme and Dumont 2008a); Brancelia (Van Damme and Sinev 2011), which corresponds to the hercegovinae-complex; and Anthalona Van Damme, Sinev \& Dumont, 2011, which includes species of the verrucosa-complex (Van Damme et al. 2011a). Most recently, Sinev (2015a) included the pulchella-group in Ovalona Van Damme \& Dumont, 2008.

Likewise, the position of $A$. dentifera is doubtful because its morphology is very different from that of the "true Alona", which is represented by the quadrangularis-group only (Van Damme and Dumont 2008b; Van Damme et al. 2010). Van Damme and Dumont (2008a) suggested that $A$. dentifera belongs to a large lineage of Alona sensu lato, named the Coronatella-branch, and that it may be close to Leberis, as evidenced by molecular tools (Elias-Gutiérrez et al. 2008) or to the Coronatella genus. Although Chatterjee et al. (2013) consider A. dentifera as a member of the Coronatella genus, this species seems to be part of a group with separate evolution, together with Alona siamensis Sinev \& Sanoamuang, 2007. Alona dentifera and A. siamensis share synapomorphies, as showed by Sinev and Sanoamuang (2007). Besides, the male morphology of $A$. dentifera is quite different from Coronatella and Leberis (see description below).

Thus, our aim is to evaluate the morphological traits of Alona dentifera, based on original material from Brazil and Argentina, and to describe the adult male, for the first time. Additionally, we relocate $A$. dentifera to a new genus, which also includes A. siamensis.

\section{Methods}

The description of the new genus was based on material collected in different localities in Brazil and Argentina (see material examined) and data from the literature (Sinev et al. 2004). The selected animals were transferred to drops of glycerol on slides and dissected under a stereomicroscope. The morphology of appendages and other structures was studied using a phase contrast microscope Olympus BX41. To enumerate the setae of limbs, we used the proposal of homology from Kotov (2000a, b), which presented stability when tested in different cladoceran groups (Kotov et al. 2010). Drawings were prepared using a camera lucida attached to a phase contrast microscope Olympus BX41. 
The following abbreviations were used in the text, table and figures:

$\begin{array}{ll}\text { A1 } & \text { antennule; } \\ \text { A2 } & \text { antenna; } \\ \text { as } & \text { accessory seta; } \\ \text { CBS } & \text { copulatory brush seta; } \\ \text { en } & \text { endite; } \\ \text { ep } & \text { epipod; } \\ \text { ex } & \text { exopod; } \\ \text { fc } & \text { filter comb; } \\ \text { gfp } & \text { gnathobasic filter plate; } \\ \text { gn } & \text { gnathobase; } \\ \text { IDL } & \text { inner distal lobe; } \\ \text { il } & \text { inner lobe; ms: male seta; } \\ \text { ODL } & \text { outer distal lobe; } \\ \text { P1-4 } & \text { limbs I-IV; } \\ \text { PA } & \text { postabdomen; } \\ \text { pep } & \text { pre-epipod; } \\ \text { s } & \text { sensillum. }\end{array}$

\section{Depository abbreviations}

FDRS Personal collection of Francisco Diogo R. Sousa;

CLLA Slides collection of the GEEA, at Universidade Católica de Brasília, Brazil;

ZMOU Zoological Museum of Oslo University;

ZMMU Zoological Museum of Moscow State University.

\section{Taxonomy}

Class Branchiopoda Latreille, 1817

Order Anomopoda Sars, 1865

Family Chydoridae Dybowsky \& Grochowski, 1894 emend. Frey, 1967

Subfamily Aloninae Dybowsky \& Grochowski, 1894 emend. Frey, 1967

\section{Magnospina gen. n.}

http://zoobank.org/8BA31D3E-9088-4642-B489-EB9DB45FE9FA

Type species of the genus. Magnospina dentifera comb. n. = Alona dentifera (Sars, 1901).

Etymology. The name "Magnospina" is derived from two Latin words, magna= large, long and spina = spine. The generic name refers to the long basal spine on the postabdominal claw. 
Description. Parthenogenetic female. Habitus without dorsal keel, ovoid or with moderate lateral compression, length $0.32-0.48 \mathrm{~mm}$, maximum height before the mid-length of body; body height/length about 1.3-.17. Head Eye and ocellus of subequal or different sizes. Rostrum short from a lateral view, wide from a frontal view, not pointed, rounded or truncated; head shield wide, with the distance between the mandibular articulations higher than length of its posterior portion, with or without ornamentation; head pores absent or presents, in last case three connected main head pores, lateral head pores minute. Labral keel wide, oval and naked, apex not elongated. Carapace ornamentation not evident, slightly punctuated or with narrow longitudinal lines; valves armed with 40-53 setae internally inserted at the ventral margin and differentiated in three groups, setae from the anterior group markedly longer than median and posterior groups; ventral margin with a distinctive rounded angle at $1 / 3$ of the length of the margin. Anteroventral corner of valves rounded; posteroventral corner armed with 1-4 large denticles, broad at their bases, protruding downwards, without setules between them; posterodorsal corner poorly defined. Posterior margin almost straight, armed with inner setules on the carapace which are not arranged in groups. Antennule not exceeding the tip of the rostrum, about 2.5 times as long as it is wide; three or four rows of setules on the antennular body. Antennular sensory seta about $1 / 2$ length of antennular body. Nine aesthetascs of different length present in a distal position not exceeding the length of antennular body. Antenna with formula of antennal setae 003/113, spines 101/001; first segment of endopod and exopod elongated, about two times longer than the others segments; weak setules or spicules on the segments. First exopod segment with a narrow, naked or plumose seta, with length similar or slightly longer than the branches. Spine on the first endopod segment longer than second endopod segment. Apical spines slightly longer than the apical segments or about two times shorter than the apical segment itself. Three plumose apical setae not differentiated in size among themselves. Postabdomen approxamately 1.3-2.5 times as long as wide, narrowing distally. Dorsal margin weakly convex or straight. Preanal angle clearly prominent; preanal, anal and postanal margins of different length; postanal margin about 1.5-1.8 longer than anal margin, armed with 9-13 marginal denticles, of which the most distal (1-4) might be individualized, proximal denticles organized in clusters; 8-10 lateral fascicles with setules relatively weak. Postabdominal claw inserted on the projection of postabdomen, 1.3-1.5 times longer than anal margin; spinules on the ventral margin may be present; pecten of spinules on the internal and external face of claw, median pecten with strong spinules; base of the claw armed with 1-5 long and strong spinules. Basal spine almost straight, remarkably long, longer than $2 / 3$ of the postabdominal claw length, with or without spinules on the dorsal margin. First Maxilla with two setulated setae. Limb I with epipod oval, with a finger-like projection. ODL with bisegmented seta, serrated from middle portion towards the distal portion; accessory seta implanted near the base of the ODL. IDL (en 4) with two robust setae (2-3), seta (1) rudimentary or absent; IDL setae 2-3 thick, armed with thick basal denticles. Endite 3 with four setae, anterior seta (1) shorter or similar in length to posterior setae $(\mathrm{a}-\mathrm{b})$; setae $(\mathrm{a}-\mathrm{b})$ of similar or different length; a sensillum might 
be present on the endite. Endite 2 with three posterior long setae (d-f) which differ strongly in length among themselves; seta (d) shorter than the seta (e), setae (e-f) with thick spinules on the lateral face; a sensillum might be present on the endite. Endite 3 armed with three posterior setae (g-i); seta (i) plumose, about 1/2 of the setae (g-h). Ejector hooks relatively short. Ventral face of the limb with six-seven groups of setules organized in clusters. Limb II with exopod elongated, short seta present which might be plumose, about two-three times shorter than exopod itself. Inner portion armed with eight scrapers not specialized and decreasing in length towards distal portion, but with some denticles on the scrapers 6-8; anterior soft setae absent; gnathobase armed with four elements, filter comb armed with seven setae, of which two proximal are shorter than the others. Limb III with pre-epipod rounded and setulated, epipod oval with a short finger-like projection. Exopod with four distal and two lateral setae; fifth and sixth setae differentiated in length, third and fourth setae long; second seta about 1.4-1.7 times longer than first setae. Setae 3-6 clearly plumose. Distal endite armed with three setae and one sensillum, setae 1-2 scraper-like of different length; third seta curved and armed with many bilaterally implanted setules (3); four plumose posterior setae present. Basal endite with four soft anterior setae increasing in length towards the gnathobase, a sensillum might be present. Gnathobase with three elements, filtercomb with seven setae. Limb IV with pre-epipod rounded or rectangular and setulated, epipod oval with a long finger-like projection. Exopod with six marginal setae; first and second setae long, not plumose; third seta plumose, short, about two times shorter than the second seta; fourth seta long and plumose; fifth and sixth setae plumose and with similar lengths; Distal endite with four setae (1-4), one scraper-like (1), three flaming-torch-like (3-4); flaming-torch setae not modified. Basal endite with three slightly setulated soft setae. Gnathobase armed with a setulated seta shorter than the length endite itself, filter comb with five setae. Limb $V$ with pre-epipod rounded or rectangular and setulated, epipod oval with a long finger-like projection. Exopod not divided in lobes, armed with four plumose setae. Setae 2-4 of subequal lengths; first seta about two-three times shorter than the other setae. Internal lobe wide, oval and with long setules apically and laterally implanted; two setulated setae on the inner face which are shorter than the length of lobe itself. Filter comb with one or without seta. Limb VI absent.

\section{Adult male. As for Magnospina dentifera comb. $\mathrm{n}$.}

Diagnosis of the genus. Parthenogenetic female. Habitus ovoid, without dorsal keel. Head with rostrum wide, not pointed; head shield wide with distance between mandibular articulations higher than length of its posterior portion, main head pores absent in adults of $M$. dentifera comb. $\mathrm{n}$. or with three connected main head pores in $M$. siamensis comb. n.; lateral head pores absent $(M$. detifera comb. n.) or present ( $M$. siamensis comb. n.). Labral keel wide and naked, apex not elongated. Carapace ornamentation not evident, punctuated or with narrow longitudinal lines; valves armed with 40-53 setae internally inserted at the ventral margin and differentiated in three groups, setae from the anterior group markedly longer than median and posterior groups; ventral margin with a distinctive rounded angle at $1 / 3$ of the margin length; 
posteroventral corner armed with 1-4 large denticles, broad at their bases, protruding downwards, without setules between them. Antennule not exceeding the tip of the rostrum, nine aesthetascs of different lengths present distally. Antenna with formula of antennal setae $003 / 113$; spines 101/001; basal segments on the exopod and endopod about two times longer than the other segments; weak setules or spicules on the segments. Postabdomen narrowing distally, preanal angle prominent; postanal margin armed with 9-13 marginal denticles which the most distal (1-4) might be individualized, proximal denticles organized in clusters; eight-10 lateral fascicles with weak setules. Postabdominal claw inserted on the projection of postabdomen, longer than anal margin; spinules on the ventral margin may be present; pecten of spinules on the internal and external face of the claw, base of claw armed with 1-5 long and spinules. Basal spine remarkably long, longer than $2 / 3$ of postabdominal claw length, with or without spinules on the dorsal margin (absent in M. siamensis comb. n.). Limb I with endite 1 armed with three setae (g-i); IDL (en 4) with two robust setae (2-3), seta 1 rudimentary ( $M$. dentifera comb. n.) or absent ( $M$. siamensis comb. n.); IDL setae 2-3 thick, armed with thick basal denticles. Limb II without soft setae; short seta on the exopod; scrapers not specialized, but with some denticles, especially on scrapers 6-8; gnathobase armed with four elements, filter comb armed with seven setae, of which two proximal are shorter than others. Limb III with six setae on the exopod, third and fourth setae long; distal endite armed with three setae and one sensillum; gnathobase with three elements, filter comb with seven setae. Limb IV relatively short, six setae on the exopod; third seta plumose, short, about two times shorter than the second seta; flaming-torch setae on the distal endite not modified; gnathobase armed with a setulated setae shorter than the length of endite itself, filter comb with five setae. Limb V relatively short, setae 3-4 of exopod subequal in length; filter comb reduced, with one short seta in $M$. dentifera comb. n. and none in M. siamensis comb. n.. Limb VI absent.

Adult male. Habitus smaller than female (Figure 27). Postabdomen strongly narrowing distally. Postabdominal claw short and ticker than female (Figures 30-31). Basal spine about half-length of postabdominal claw, with tip forked (Figure 31). Limb I with two setae on the IDL (en4), setae armed with denticles; male seta with tip slightly curved; copulatory hook with one projection on the tip (Figures 32-33).

Differential diagnosis. The synapomorphies of Magnospina gen. n. are (1) basal spines longer than $2 / 3$ of postabdominal claw, (2) presence of $1-4$ large denticles, broad at their bases, protruding downwards, without setules between them. Magnospina gen. n. can also be differentiated from the genus Coronatella because it has a distinctive rounded angle at $1 / 3$ of the length of the ventral margin, marginal setae of valves differentiated in three groups, setae from anterior group markedly longer, wide rostrum, postabdomen narrowing distally; the males of Coronatella do not bear two lateral aesthetascs on the antennules. Magnospina gen. n. differs from Anthalona in the presence of a distinctive rounded angle at $1 / 3$ of the length of the ventral margin, sacks underneath lateral head pores (cosmaria) being absent, shape of postabdomen, poorly developed setules of the lateral fascicles, morphology of IDL (which does not have specialized denticles), and armature of limb I; the males of Anthalona also do not 
bear lateral aesthetascs on the antennules. The new genus differs from Karualona in the morphology of IDL setae, shape of the postabdomen, poorly developed setules of the lateral fascicles, seta on the exopod of limb II (present in Magnospina gen. n. and absent in Karualona) and endite basal of limb IV armed with three flaming-torch; the antennule of Karualona males bear just one lateral aesthetasc. Magnospina gen. n. is closer to Leberis according to Eliáz-Gutiérrez et al. (2008); however, it is distinguished by the presence of long setae on anterior group of ventral margin of the carapace, in the morphology of setae 2-3 of the IDL (Figures 18-19), presence of seta on exopod of limb II, absence of a dorsal keel (Figures 1-5), and presence of a long basal spine on postabdominal claw of postabdomen (Figures 13-16); males of Leberis also do not bear denticles on the posteroventral corner of valves. Magnospina gen. n. differs from Celsinotum Frey, 1991 in the absence of a dorsal keel, absence of spine-like setae on the posterior portion of valves, presence of relatively long apical (endopod and exopod) and basal (endopod) spines on the segment of the antenna, long basal spine on the postadbominal claw, absence of a rudimentary seta (i) on endite 1 of limb I (in Magnospina gen. $\mathrm{n}$. setae (i) is developed). The new genus differs clearly from Bergamina Elmoor-Loureiro, Santos-Wisniewski \& Rocha, 2013 in morphology of postabdomen, presence of denticles on the posteroventral margin of valves and absence of anterior seta between endites 1-2 of limb I (see Elmoor-Loureiro et al. 2013). Magnospina gen. n. differs from Extremalona Sinev \& Shiel, 2012 in general morphology, presence of denticles on the posteroventral margin of valves, postabdomen morphology and armature of setae 2-3 of IDL; male of Extremalona also bears six lateral aesthetascs on the antennules. Ovalona Van Damme \& Dumont, 2008 has a well-developed seta 1 on the IDL, endite 1 of the limb I without seta (i) and exopod of the limb III armed with seven setae. Magnospina gen. n. does not present any of aforementioned morphological traits to Ovalona. Table 1 shows the main differences and similarities between genera of the Coronatella-branch.

\section{Magnospina dentifera (Sars, 1901), comb. n.}

Figures 1-33

Alona dentifera (Sars, 1901): Sinev et al. 2004: 101, 103-104, figures 1-39; Güntzel et al. 2010: 95, table 1; Sousa and Elmoor-Loureiro 2012: 356, table 2; DebastianiJúnior et al. 2015: 24, table 2.

Alona broaensis: Matsumura-Tundisi and Smirnov 1984: 327-328, figures 15-21;

Güntzel et al. 2010: 95, table 1; Debastiani-Júnior et al. 2015: 24, table 2.

Type locality. "neighborhood of São Paulo", State of São Paulo, Brazil.

Material type. Lectotype: Parthenogenetic $O$, ZMOU F12341a, selected by D. Frey. Paralectotype: 5 parthenogenetic $q 9$, ZMOU F12341b; 1 parthenogenetic $q$ F12341c; 4 parthenogenetic $q$, , I instar juvenile 9 , ZMOU F12386g; 2 instar II juvenile + ZMOU F12386q; 2 partenogenetic $ᄋ+q$, instar II juvenile \#, ZMOU, slide 


\begin{tabular}{|c|c|c|c|c|c|c|c|c|c|c|c|c|}
\hline 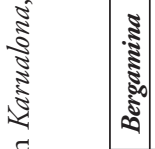 & & $\begin{array}{l}\infty \\
0 \\
0 \\
0\end{array}$ & 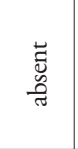 & 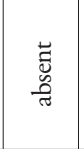 & $\begin{array}{l}\vec{z} \\
\bar{z} \\
\bar{\Xi} \\
\bar{g}\end{array}$ & 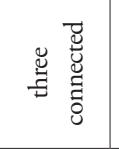 & 芞 & 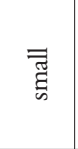 & 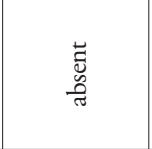 & 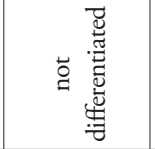 & 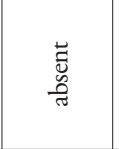 & 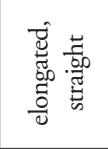 \\
\hline 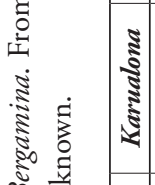 & & $\begin{array}{l}f \\
f \\
0 \\
f \\
0\end{array}$ & $\begin{array}{l}\text { च्ّ } \\
\text { है } \\
\text { ते }\end{array}$ & $\begin{array}{l}\tilde{\breve{J}} \\
\text { त्र }\end{array}$ & $\begin{array}{l}\vec{z} \\
\bar{v} \\
\bar{\Xi} \\
\bar{a}\end{array}$ & 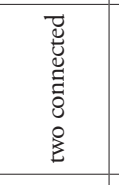 & 节 & $\begin{array}{l}\text { 品 } \\
\text { తn }\end{array}$ & 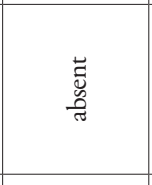 & 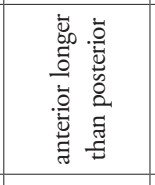 & 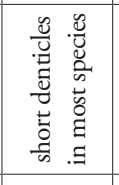 & 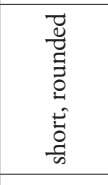 \\
\hline 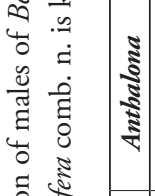 & & $\begin{array}{c}+ \\
0 \\
m \\
0 \\
0\end{array}$ & 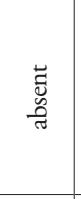 & 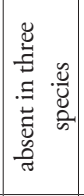 & $\frac{\vec{z}}{\vec{z}}$ & 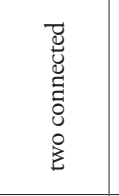 & 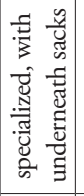 & 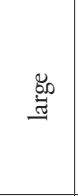 & $\begin{array}{l}\text { 苟 } \\
\text { 产 }\end{array}$ & 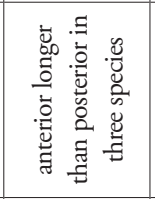 & 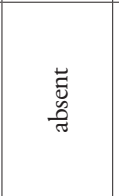 & 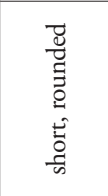 \\
\hline 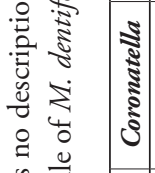 & & 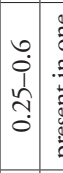 & 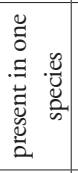 & 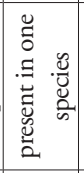 & $\frac{\vec{v}}{\vec{z}}$ & 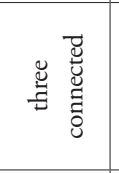 & 节 & 若 & $\begin{array}{l}\text { च. } \\
\text { त्र } \\
\text { त्र }\end{array}$ & 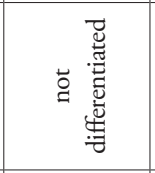 & 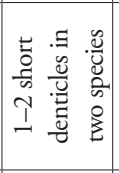 & 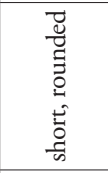 \\
\hline 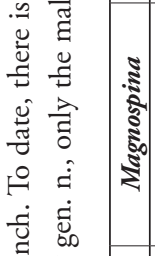 & & 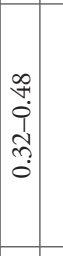 & 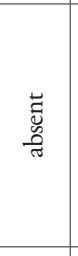 & 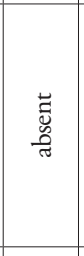 & 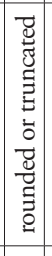 & 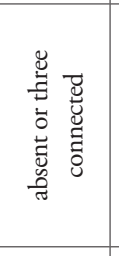 & 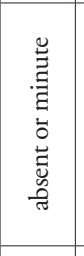 & 若 & 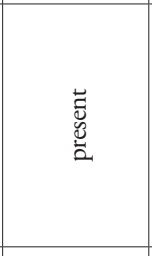 & 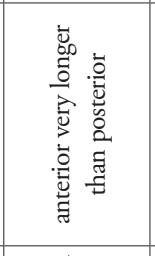 & 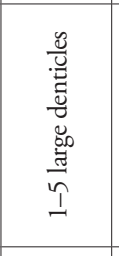 & 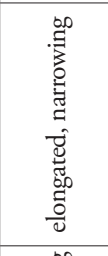 \\
\hline 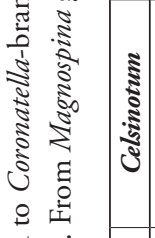 & & $\begin{array}{c}\hat{0} \\
\hat{2} \\
\hat{0}\end{array}$ & 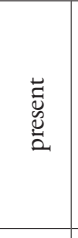 & 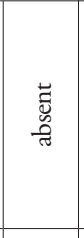 & 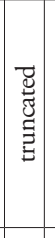 & 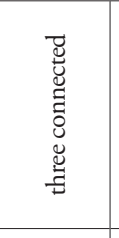 & 节 & 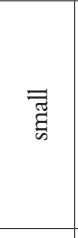 & 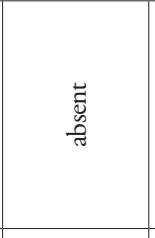 & 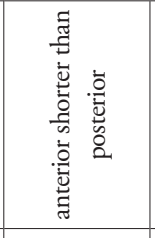 & 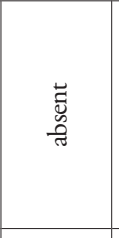 & 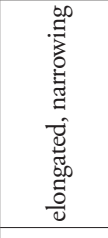 \\
\hline 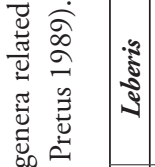 & & $\begin{array}{l}-1 \\
\overrightarrow{1} \\
\tilde{0} \\
0\end{array}$ & $\begin{array}{l}\text { 苛 } \\
\text { 岂. }\end{array}$ & 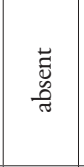 & $\begin{array}{l}\overrightarrow{\widetilde{Z}} \\
\widetilde{\Xi} \\
\mathrm{E} \\
\mathrm{E}\end{array}$ & 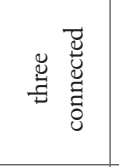 & 莺 & 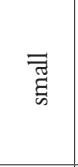 & 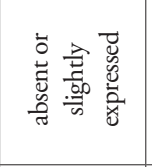 & 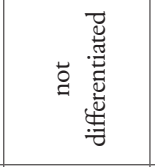 & 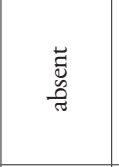 & 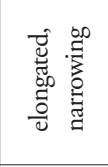 \\
\hline 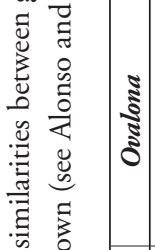 & & $\begin{array}{l}\hat{0} \\
0 \\
\hat{n} \\
\tilde{0}\end{array}$ & 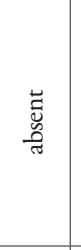 & 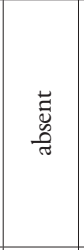 & 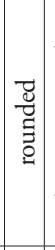 & 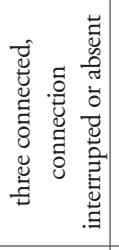 & 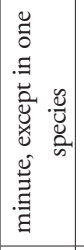 & 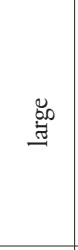 & 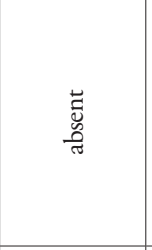 & 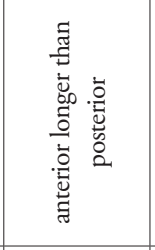 & 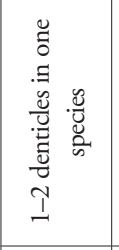 & 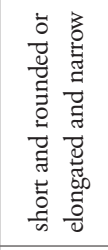 \\
\hline $\mid$ & & $\tilde{o}$ & 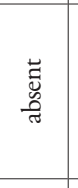 & 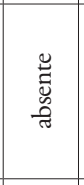 & $\left|\begin{array}{l}\vec{z} \\
\bar{v} \\
\bar{g} \\
\bar{g}\end{array}\right|$ & 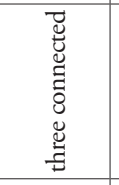 & 莺 & $\begin{array}{l}\text { 品 } \\
\text { ] }\end{array}$ & 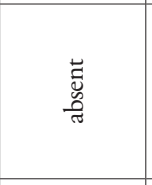 & 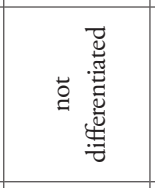 & 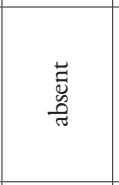 & 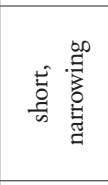 \\
\hline 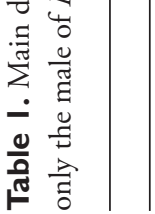 & 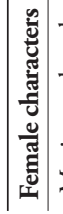 & 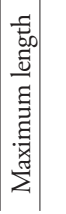 & 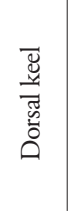 & 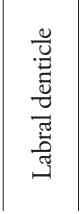 & 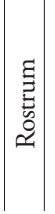 & 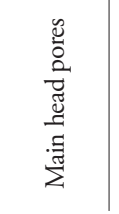 & 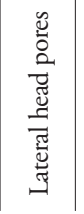 & 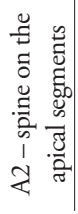 & 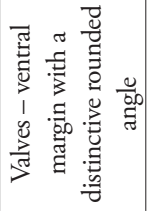 & 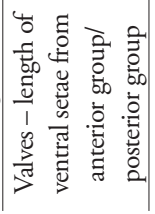 & 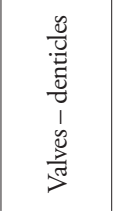 & 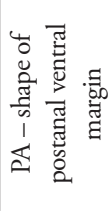 \\
\hline
\end{tabular}




\begin{tabular}{|c|c|c|c|c|c|c|c|c|c|c|c|c|c|}
\hline ט. & $\frac{\overrightarrow{0}}{\frac{0}{n}}$ & 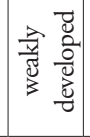 & 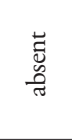 & 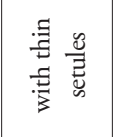 & $\begin{array}{l}\text { 䔍 } \\
\text { 岂. }\end{array}$ & 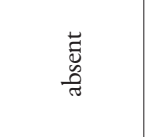 & . & 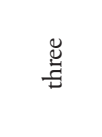 & & 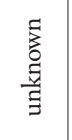 & $\begin{array}{l}\frac{5}{5} \\
\frac{5}{5} \\
\end{array}$ & 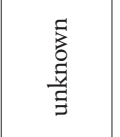 & 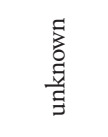 \\
\hline בิ & 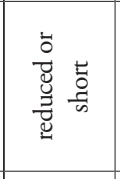 & 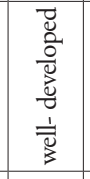 & 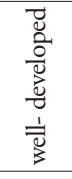 & 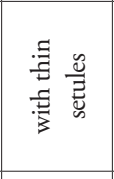 & 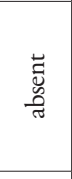 & 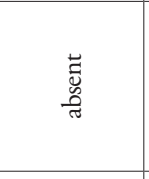 & $\frac{x}{w}$ & $\stackrel{\beta}{\xi}$ & & ๘̆ & 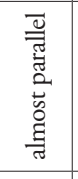 & 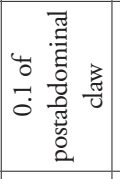 & 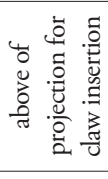 \\
\hline 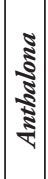 & 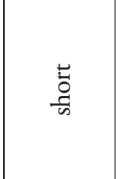 & 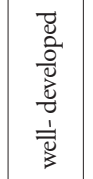 & 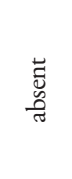 & 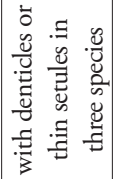 & 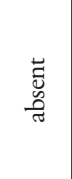 & 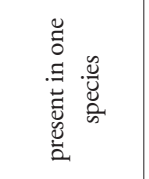 & .x & 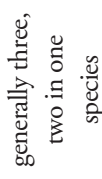 & & 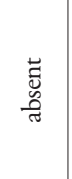 & 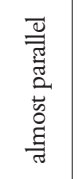 & 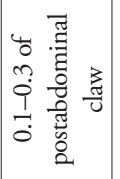 & 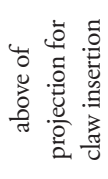 \\
\hline 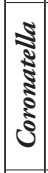 & 营 & 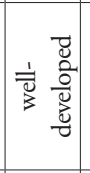 & 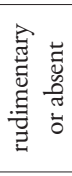 & 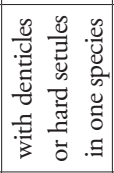 & 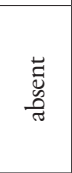 & 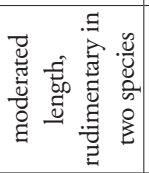 & . & 苛 & & $\begin{array}{l}\text { चू̆ } \\
\text { है }\end{array}$ & 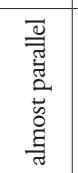 & 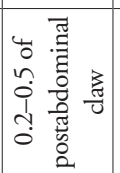 & 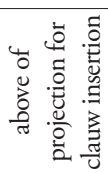 \\
\hline | & 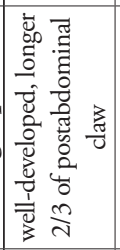 & 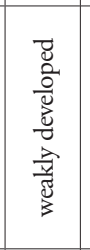 & 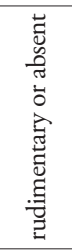 & 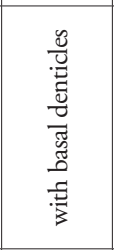 & $\begin{array}{l}\text { चूँ } \\
\text { त्त }\end{array}$ & $\begin{array}{l}\frac{\overrightarrow{0}}{-5} \\
\frac{0}{n}\end{array}$ &.$\frac{x}{w}$ & 节 & & $\xi$ & . & 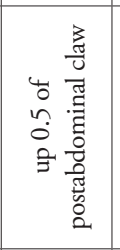 & 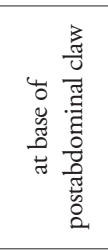 \\
\hline ปี & 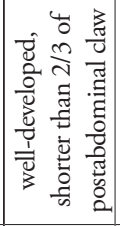 & 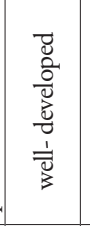 & 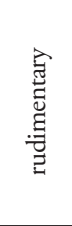 & 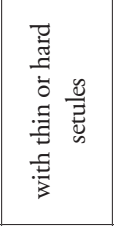 & 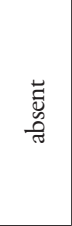 & $\begin{array}{l}\text { ज्ञ } \\
\text { त्त }\end{array}$ & . & 苞 & & 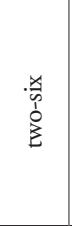 & . & 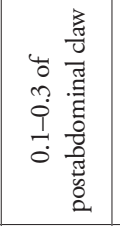 & 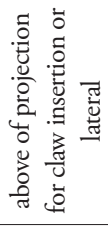 \\
\hline 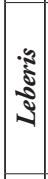 & $\begin{array}{l}\text { to } \\
\text { 总 }\end{array}$ & 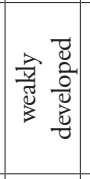 & 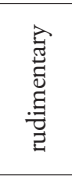 & 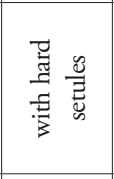 & $\begin{array}{l}\text { Ŭ } \\
\text { 岇 }\end{array}$ & $\begin{array}{l}\text { ज्ञّ } \\
\text { त्त }\end{array}$ &.$\frac{x}{m}$ & 苂 & & $\xi$ & 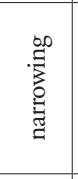 & 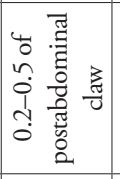 & 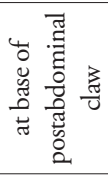 \\
\hline పే & $\begin{array}{l}\frac{t}{0} \\
\frac{\pi}{\infty}\end{array}$ & 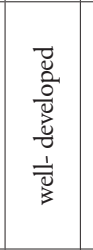 & 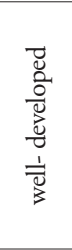 & 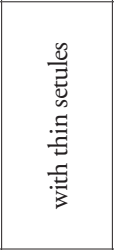 & $\begin{array}{l}\overrightarrow{\tilde{J}} \\
\text { त्र }\end{array}$ & 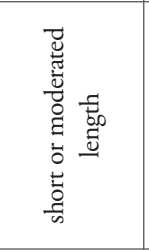 & 芯 & 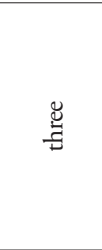 & & 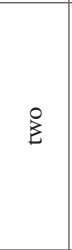 & 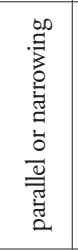 & 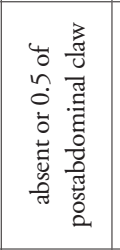 & 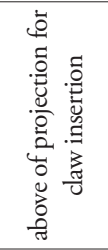 \\
\hline 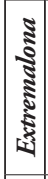 & $\begin{array}{l}\frac{t}{0} \\
\frac{d}{n}\end{array}$ & 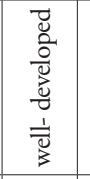 & 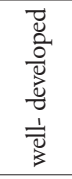 & 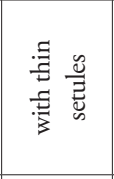 & 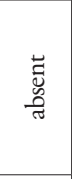 & 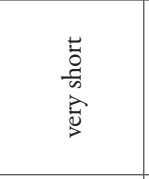 & : & 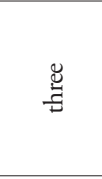 & & : & 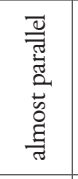 & 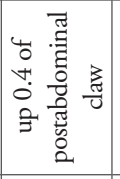 & 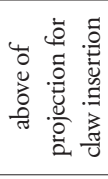 \\
\hline & 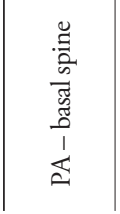 & 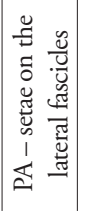 & 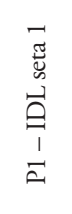 & 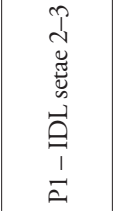 & 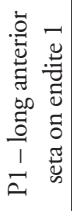 & 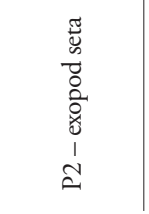 & 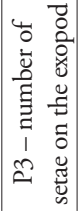 & 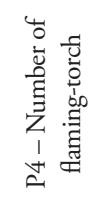 & 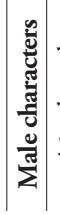 & 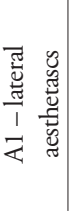 & 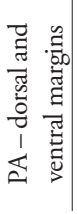 & 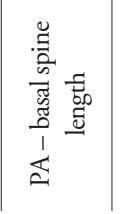 & 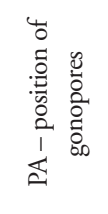 \\
\hline
\end{tabular}




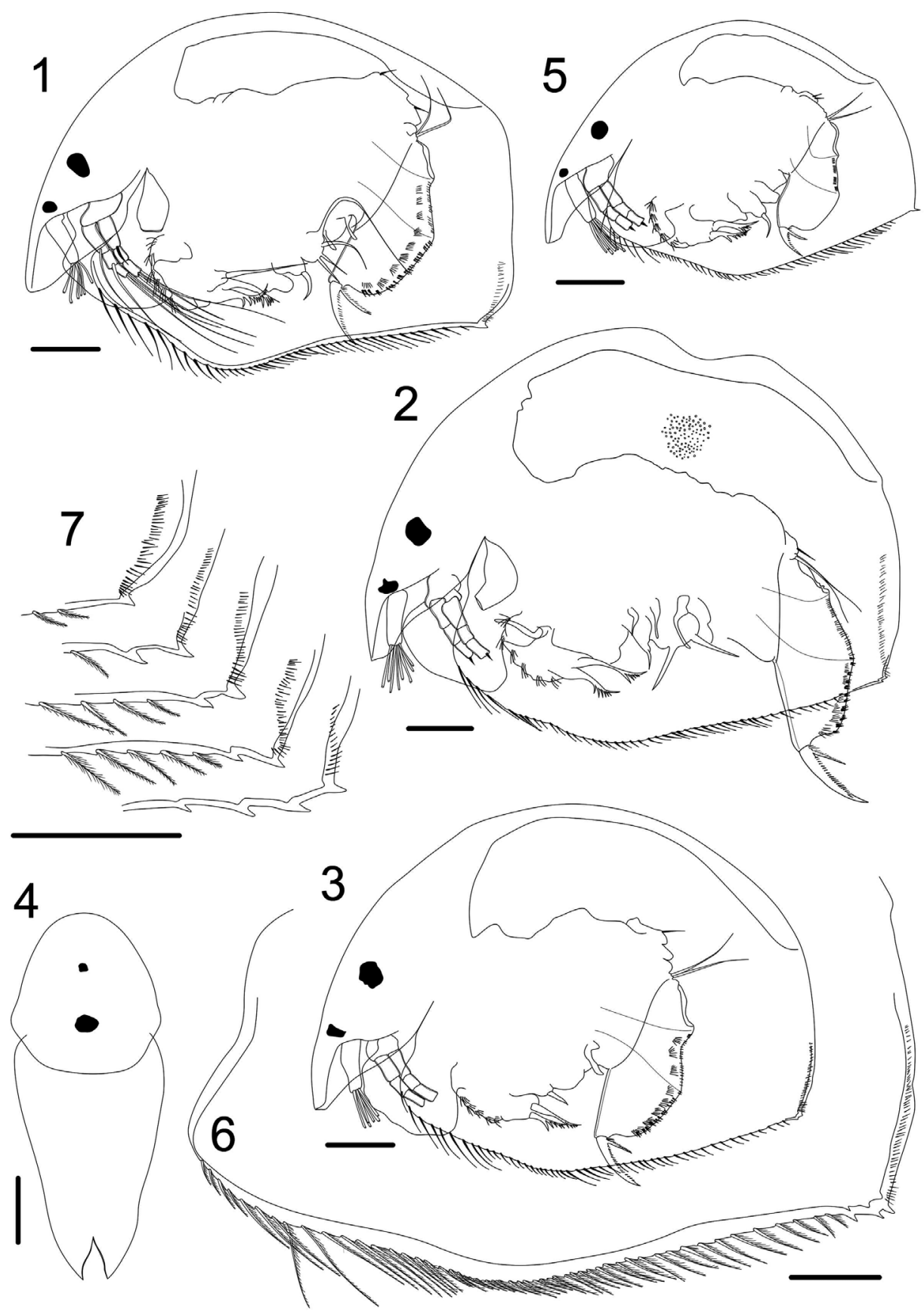

Figures I-7. Magnospina dentifera comb. n., parthenogenetic female. I habitus from Pantanal, Mato Grosso do Sul, Brazil 2 habitus from São Paulo, Brazil 3-4 habitus from San Pedro, Argentina parthenogenetic female adult from $\mathbf{5}$ habitus, parthenogenetic female juvenile from San Pedro, Argentina $\mathbf{6}$ ventral margin of carapace from Distrito Federal, Brazil 7 denticles on the posteroventral margin of carapace. Scale bars: $50 \mu \mathrm{m}$. 


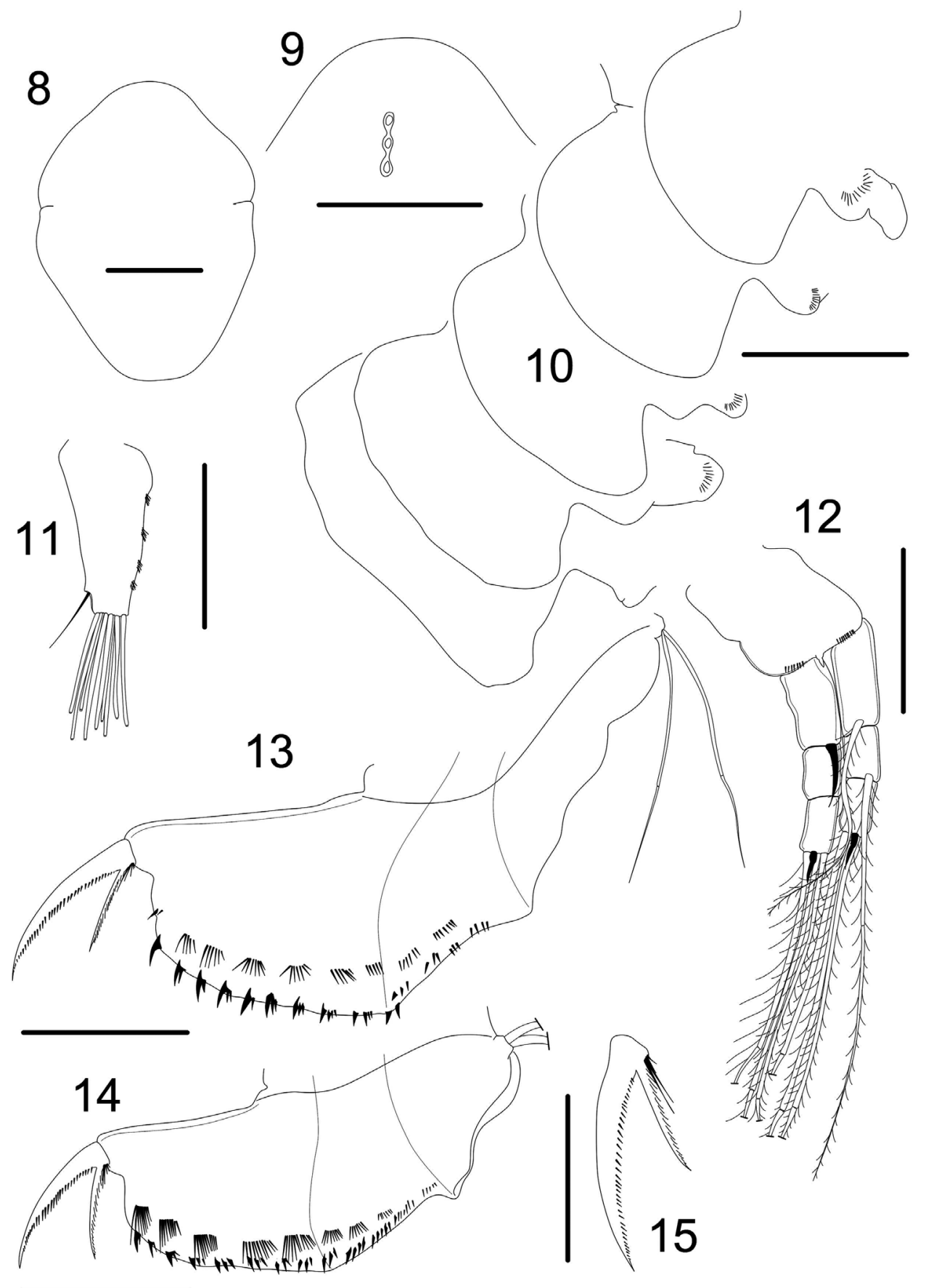

Figures 8-15. Magnospina dentifera comb. n., parthenogenetic females. 8 head shield 9 main head pores, female juvenile $\mathbf{I} \mathbf{0}$ labral kell II antennule $\mathbf{I} \mathbf{2}$ antenna $\mathbf{3} \mathbf{3} \mathbf{|} \mathbf{4}$ postabdomen $\mathbf{5}$ postabdominal claw. Scale bars: $50 \mu \mathrm{m}$. 
F9130; 6 parthenogenetic $9+$, ephippial $\odot$, ZMOU, slide F9131; 2 parthenogenetic 우오, ephippial ㅇ, ZMOU, slide F9131.

Material examined. Nine parthenogenetic females and one adult male from Henrique pond, Brasília National Park, Distrito Federal, Brazil (1541'18"S; 47056'26.10"W), material collected by Grupo de Estudos de Ecossistemas Aquáticos (GEEA) in ix.2009 (FDRS048). One parthenogenetic female from Henrique pond, Brasília National Park, Distrito Federal, Brazil (1541'16.5"S; 4756'22.2"W), material collected by Lourdes M. A. Elmoor-Loureiro on 27.v.2002 (FDRS049). One parthenogenetic female from Cabocla II pond, Campo de Instrução de Formosa, Goi-

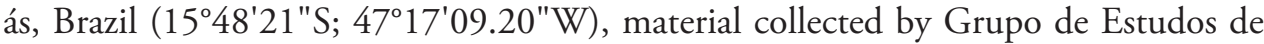
Ecossistemas Aquáticos (GEEA) on viii.2009 (FDRS050). Six adult parthenogenetic females and one juvenile from Baía da Célia, Fazenda Nhumirim (18 $59^{\circ} 27.5^{\prime \prime}$, $56^{\circ} 39^{\prime} 41.0^{\prime \prime W), ~ P a n t a n a l, ~ M a t o ~ G r o s s o ~ d o ~ S u l, ~ B r a z i l, ~ m a t e r i a l ~ c o l l e c t e d ~ o n ~ 07 . i x .2000 ~}$ by Valéria Barros. Four parthenogenetic females from Criminosa Pond $\left(21^{\circ} 40^{\prime} 28.8^{\prime \prime S}\right.$, $57^{\circ} 53$ '28.5"W) identified as Alona broaensis, Porto Murtinho, Pantanal, Mato Grosso do Sul, Brazil, material collected on 19.i.2010, leg Adriana Maria Güntzel (FDRS054). Two parthenogenetic females from Coqueiral Pond, Paranapanema River, Angatuba, São Paulo, Brazil (2329'22.64"S; 48 37'6.65"W). Material collected by Lourdes M. A. Elmoor-Loureiro on 30.v.2001 (CLLA063, 65-66). Two parthenogenetic females from Esquina, Middle Paraná River, Argentina (3000.54'59"S; 59³2'51.93"W). Material collected by José Roberto Debastiani Júnior on 12.vi.2010 (FDRS052). Six parthenogenetic females from San Pedro, Lower Paraná River, Argentina (3040'49"S; $\left.59^{\circ} 18^{\prime} 48.80^{\prime W}\right)$. Material collected by José Roberto Debastiani Júnior on 14.vi.2010 (FDRS053). Three parthenogenetic females from Pimenteira pond, Mata da Pimenteira State Park, Serra Talhada, Pernambuco, Brazil (753'48.96"S, 38¹8'14.30"W). Material collected by Leidiane Pereira Diniz on 13.iv.2014 (FDRS407).

Differential diagnosis. Magnospina dentifera comb. n. differs from Magnospina siamensis comb. $\mathrm{n}$. because it has a rounded and wide rostrum and main and lateral head pores are absent in adult. Apical spines of the antenna about two times shorter than the apical segments. On the limbs, the main differences are: $M$. dentifera comb. n. bears a rudimentary seta 1 on the IDL, setae 2-3 of IDL armed with at least seven denticles and the presence of one seta on the filter comb of limb V.

Diagnosis. Habitus ovoid, without dorsal keel, not compressed laterally, length $0.32-0.48 \mathrm{~mm}$; eye and ocellus of different sizes. Head with rostrum wide, rounded, not pointed; head shield wide, with broadly rounded posterior margin, distance between mandibular articulations higher than length of its posterior portion, main head pores absent in adults, two or three connected main head pores in juveniles (Figures 8-9); lateral head pores absent. Labral keel wide, large and naked, apex not elongated (Figure 10). Carapace ornamentation slightly punctuated or not evident; ventral margin of carapace with a distinctive rounded angle at $1 / 3$ of length; valves armed with 40-53 setae internally inserted at the valve ventral margin and differentiated in three groups, setae from anterior group markedly longer than median and posterior groups (Figures 1-6); posteroventral corner armed with 1-4 large denticles, broad at their 
bases, protruding downwards, without setules between them (Figure 7). Antennule do not exceed the tip of the rostrum, nine apical aesthetascs of different lengths which do not exceed the length of the antennular body (Figure 11). Antenna with formula of antennal setae 003/113, spines 101/001; first segment of endopod and exopod elongated; weak setules or spicules on the segments; spine on the first segment of the endopod longer than second segment length; apical spines about two times shorter than the apical segments (Figure 12). Postabdomen narrowing distally, length about 1.3 times its height; preanal angle prominent; postanal margin about 1.5 times longer than the anal margin armed with 10-13 groups of denticles, 1-3 most distal denticle might be individualized; 8-10 lateral fascicles armed with weak setules (Figures 13-14). Postabdominal claw inserted on the projection of postabdomen, longer than anal margin; spinules on the ventral margin may be present; pecten of spinules on the internal and external face of the claw, base of claw armed with 1-5 long and strong spinules (Figures 14-15). Basal spine remarkably long, longer than $2 / 3$ of postabdominal claw length, with spinules on the dorsal margin (Figures 14-15). Limb I with IDL (en 4) armed with one rudimentary seta (1) and two well-developed setae (2-3) which bears at least seven distinguishable denticles, basal denticles thick; seta (1) on endite 3 shorter than setae (a-c), setae (a-b) of different length, endite 3 with an element; endite 2 armed with three setae (d-f), element present; endite 1 with three setae (g-i) (Figures 16-19). Limb II without anterior soft setae; seta on the exopod short, slightly plumose; scrapers not specialized, but with some denticles, especially on scrapers 6-8; gnathobase armed with four elements, filter comb armed with seven setae, of which two are shorter than others (Figure 20). Limb III with six setae on the exopod, third and fourth setae long; third seta longer than the second seta; distal endite armed with three setae and one sensillum; gnathobase with three elements, filter comb with seven setae (Figures 21-22). Limb IV relatively short, six setae on the exopod; setae 1-2 of different lengths, flaming-torch setae on the distal endite not modified with weak setules; gnathobase armed with a setulated seta shorter than the length of endite itself, filter comb with five setae (Figures 23-26). Limb $V$ relatively short, setae 3-4 of exopod similar in length; filter comb reduced with one short seta (Figures 25-26). Limb VI absent.

Ephippial female. Not studied.

Adult male. Habitus smaller than female (Figure 27). Postabdomen strongly narrowing distally. Postabdominal claw short and ticker than female (Figures 30-31). Basal spine about half-length of postabdominal claw, with tip forked (Figure 31). Limb I with two setae on the IDL (en4), setae armed with denticles; male seta with tip slightly curved; copulatory hook with one projection on the tip (Figures 32-33).

Description of adult male. Habitus ovoid, smaller than that in female, length about $0.35 \mathrm{~mm}$, maximum height in the middle of the body (Figure 27). Head with rostrum elongated, not blunt, main head pores absent (Figure 27). Carapace without ornamentations; ventral margin with a distinctive rounded angle at $1 / 2$ of the margin length, margin armed with about 37 setae, posteroventral corner with two large denticles, broad at their bases, without setules between them (Figure 27). Antennule not exceeding the tip of rostrum, about 2.5 times as long as it is wide, with three rows 


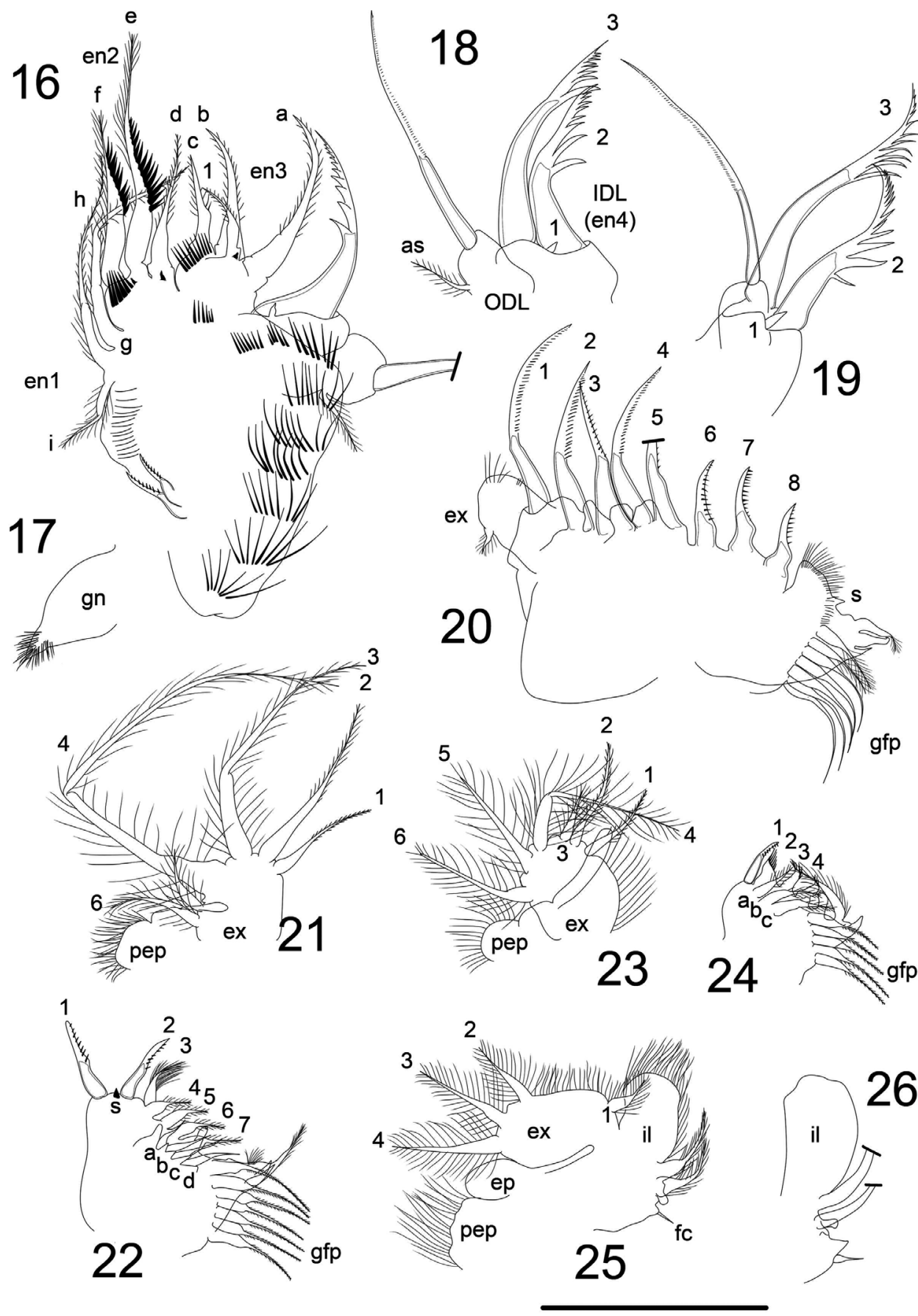

Figures 16-26. Magnospina dentifera comb. n., adult parthenogenetic females. 16 limb I 17 limb I, gnathobase 18-19 limb I, IDL and ODL 20 limb II 2 I limb III, exopod 22 limb III, endites 23 limb IV, exopod $\mathbf{2 4}$ limb IV, endites $\mathbf{2 5}$ limb V 26 limb V, internal lobe. Scale bars: $50 \mu \mathrm{m}$. 


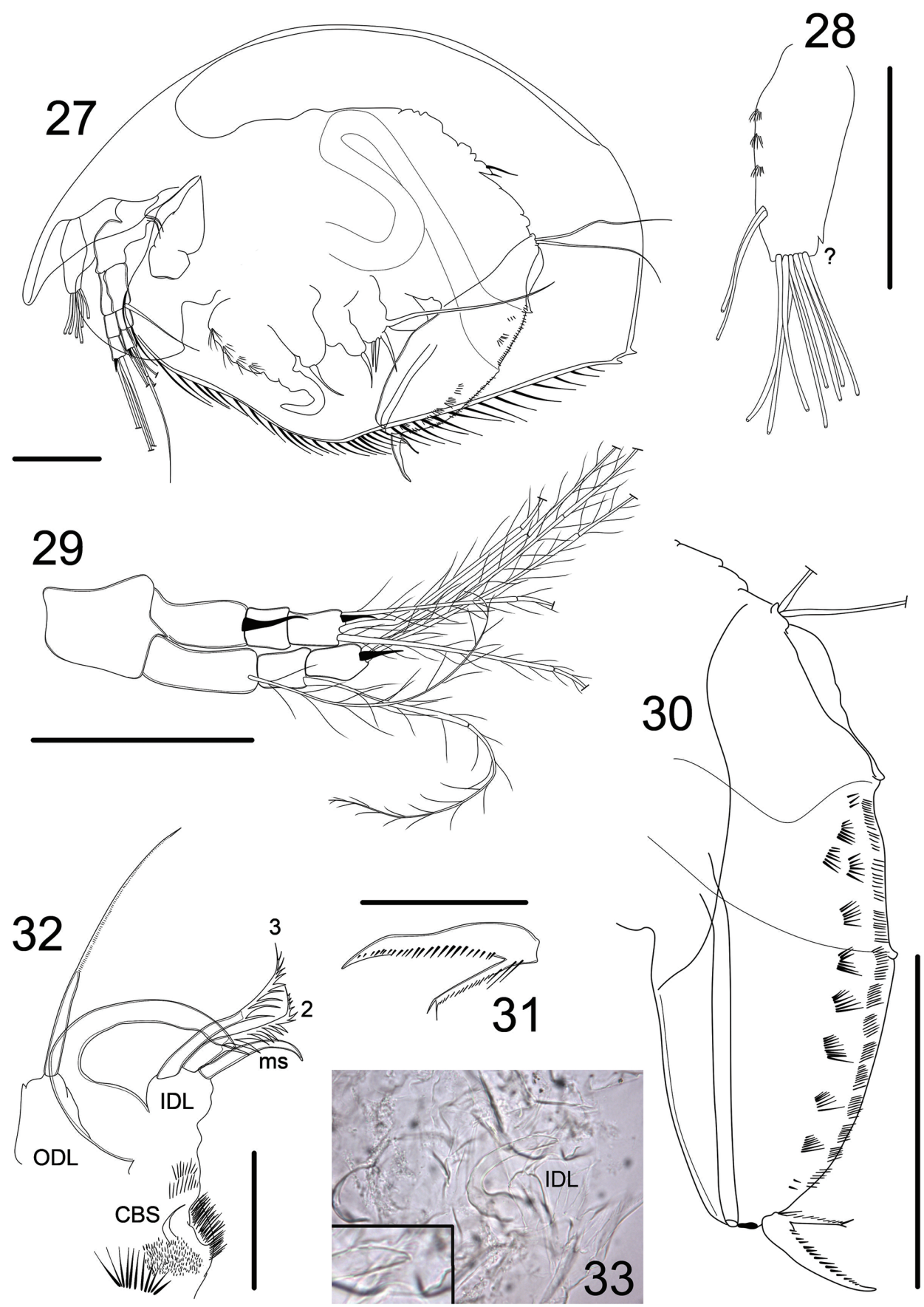

Figures 27-33. Magnospina dentifera comb. n., adult male from Henrique Pond, Brasília National Park, Distrito Federal, Brazil. $\mathbf{2 7}$ habitus $\mathbf{2 8}$ antennule $\mathbf{2 9}$ antenna $\mathbf{3 0}$ postabdomen $\mathbf{3 1}$ postabdominal claw 32 limb I, IDL and ODL 33 limb I, copulatory hook. Scale bars: $50 \mu \mathrm{m}(\mathbf{2 7 - 3 0 )} ; 25 \mu \mathrm{m}(\mathbf{3}$ I-33). 
of short setules on body antennular; eleven aesthetascs, two lateral and nine apical ones. Sensory seta and male seta not studied (Figures 27-28). Antenna as described for females, however, apical spines relatively longer (Figure 29). Postabdomen as long as in female, strongly narrowing distally. Anal margin shorter than postanal margin; 12 rows of thin setules on the anal and postanal margin; eight lateral fascicles with weak setules of which do not exceed postanal margin (Figure 30). Postabdominal claw smaller and more robust as comapared with female, base armed with long and strong spinule, pecten armed with strong spinules at the median portion of the claw (Figures 30-31). Basal spine long, about half-length of postabdominal claw, with a forked tip, ventral margin armed with spinules (Figure 31). Limb I with copulatory hook curved, U-shaped, projection at the tip present, copulatory brush seta shorter than male seta on IDL (en4), the latter armed with three setae; male setae thick with tip slightly curved; setae 2-3 armed with proximal denticles (as observed in female); ODL seta longer than IDL setae (Figures 32-33).

Distribution. Neotropics, from Southern U.S.A to Argentina (Sinev et al. 2004).

\section{Magnospina siamensis comb. $\mathbf{n}$.}

Alona siamensis: Sinev and Sanoamuang 2007: 145, 147-148, figures 1-30; Van

Damme and Sinev 2013: 226-228; Korovinchinsky 2013: 114, 119, tables 1-2. Coronatella dentifera (Sars, 1901): Chartejee et al. 2013: 43.

Type locality. Rice field at Ban Bayao Baghe Sub-district, Phannanichom District, Sakhonnakhon Province, Thailand, 01.09.2004.

Material type. Holotype: parthenogenetic female, ZMMU, MI-73. Paratypes: 2 parthenogentic females, ZMMU, MI74.

Differential diagnosis. Magnospina siamensis comb. n. differs from Magnospina dentifera comb. $\mathrm{n}$. because it has a truncated rostrum, three connected main head pores, minute lateral head pores and a prominent sculpture on the carapace. Apical spines of the antenna are longer than the apical segments. On the limbs, the main differences are: IDL is armed with two setae (2-3), seta 2 with two thick basal denticles, seta 3 with one thick basal denticle, limb V without filter comb.

Diagnosis. Female. According to the literature (Sinev and Sanoamuang 2007).

Habitus without dorsal keel, moderately compressed laterally, length $0.35-0.42$ $\mathrm{mm}$; eye and ocellus of subequal sizes. Head with rostrum wide, truncated; head shield ornamented with longitudinal lines, wide, posterior margin broadly rounded, distance between mandibular articulations higher than length of its posterior portion, three connected main head pores, lateral head pores minute. Labral keel wide, oval and naked, apex not elongated. Carapace covered with narrow longitudinal lines; ventral margin of carapace with a distinctive rounded angle at $1 / 3$ of length; valves armed with 45 setae internally inserted at the ventral margin and differentiated in three groups, setae from anterior group markedly longer; posteroventral corner armed with 2-3 
large denticles, broad at their bases, protruding downwards, without setules between them. Antennule do not exceed the tip of the rostrum, nine apical aesthetascs of different length which do not exceed the length of the antennular body. Antenna with formula of antennal setae 003/113, spines 101/001; first segment of endopod and exopod elongated; weak setules or spicules on the segments; spine on the first segment of the endopod longer than the second segment; apical spines longer than the apical segments. Postabdomen narrowing distally, length about 2.5 it is height; preanal angle prominent; postanal margin about 1.7-1.8 times longer than the anal margin armed with 3-4 single most distal denticles and 5 cluster of denticles; About 10 lateral fascicles with weak setules. Postabdominal claw inserted on the projection of postabdomen, longer than anal margin; base of claw armed with 1-2 long and strong spinules. Basal spine remarkably long, longer than $2 / 3$ of postabdominal claw length, without spinules on the dorsal margin. Limb I with two thick setae (2-3) on the IDL (en 4), seta (2) with two thick basal denticles, seta (3) with one thick basal denticle; setae (1, a-c) on the endite 3 of subequal length, endite 3 without element; endite 2 armed with three setae (d-f), without element; endite 1 with three setae (g-i). Limb II without anterior soft setae; seta on the exopod short, not plumose; scrapers not specialized, but with some denticles, especially on scrapers 6-8; gnathobase armed with four elements, filter comb armed with seven setae, of which two are shorter than the others. Limb III with six setae on the exopod, third and fourth setae long; third seta slightly shorter than the second seta; distal endite armed with three setae and one sensillum; gnathobase with three elements, filter comb with seven setae. Limb IV relatively short, six setae on the exopod; setae 1-2 of similar lengths; flaming-torch setae on the distal endite not modified, setules on the first flaming-torch relatively longer that one observed in setae 2-3; gnathobase armed with a setulated seta shorter than length of endite itself, filter comb with five setae. Limb $V$ with setae 3-4 of exopod similar in lengths; filter comb absent. Limb VI absent.

Ephippial female and male. Unknown.

Distribution. Malysia, Thailand (Sinev 2007; Van Damme and Sinev 2013; Korovinchinsky 2013) and probably India (Chartejee et al. 2013).

\section{Discussion}

\section{Morphological analyses}

In the redescription of Alona dentifera, Sinev et al. (2004) suggested that specific morphological traits observed in this species were not enough to create a new genus, however, the description of A. siamensis (Sinev and Sanoamuang 2007) showed a new perspective about dentifera-group. Thus, separation of Magnospina gen. n. is mainly supported by such characters as: (1) basal spines longer than $2 / 3$ of postabdominal claw, (2) presence of 1-4 large denticles, broad at their bases, protruding downwards, without setules between them. Other specific morphological traits also are observed 
in Magnospina gen. n.: presence of a distinctive rounded angle at $1 / 3$ of the length of ventral margin of carapace, setae on the valves differentiated in three groups with the anterior group markedly longer than median and posterior groups, prominent preanal angle at postabdomen, setae 2-3 of IDL armed with basal denticles, six setae on limb III, and absent limb VI. The presence of six setae on exopod of limb III and absence of limb VI may be considered as simplesiomorphies of the clade Magnospina dentiferal M. siamensis, and ancestral state for the Coronatella-branch.

The morphology of head shield, main head pores and of some structures of the limbs are different between $M$. dentifera comb. n. and $M$. siamensis comb. n.; however, analogous variation in these structures was already observed in Euryalona (Rajapaksa and Fernando 1987). Species-groups of Alona sensu lato, such as the costata-group (Sinev 1999b, 2001a, 2008, Van Damme and Eggermont 2011, Van Damme et al. 2011b), verrucosa-group (Van Damme et al. 2011 a, Sinev and Kotov 2012), rectangula-group (Van Damme and Dumont 2008b, Sousa et al. 2015a), and pulchella-group (Sinev 2001b, c, 2009, Sinev and Silva-Briano 2012, Van Damme et al. 2013, Sousa et al. 2015b) also have differences in structures on head, postabdomen, and limbs.

Recently, Sinev (2014, 2015b) reviewed the morphology of Camptocercus Baird, 1843 species and showed significant differences in structures on the limbs among different species of this genus. In the same way, Celsinotum also has many differences in the morphology of head shield, postabdomen and limbs (Frey 1991, Sinev and Elmoor-Loureiro 2010, Sinev and Kotov 2012). This endorses our conclusion that the differences between $M$. dentifera comb. n. and $M$. siamensis comb. $\mathrm{n}$. should be considered at a specific level in the dentifera-group (also suggested by Sinev and Sanoamuang 2007). For Van Damme and Sinev (2013), this small lineage may represent an ancient vicariant divergence, presenting currently an Amphi-Pacific distribution, i.e. keeping in mind an antiquity of the cladoceran taxa of different ranks (Frey 1987; Kotov and Taylor 2011). Aforementioned differences in the morphology between $M$. dentifera comb. n. and M. siamensis comb. n. may be the result of adaptations to different environmental pressure on a micro-scale.

The trend in morphological radiation in the clade Magnospina gen. n. concerns the external morphology but not to features of the trunk limbs (such as in the pulchellagroup). It has been observed that a wide rostrum and the maintenance of primitive ovoid body shape, shared with other species-groups, possibly result from convergence or parallelism (Sinev et al. 2005, Van Damme and Dumont 2008b, Sinev et al. 2009, Van Damme and Sinev 2011). Regarding the limbs, an exception to the aforementioned trend seems be the armature of the IDL setae, which is more specialized in $M$. siamensis comb. $\mathrm{n}$. when compared to $M$. dentifera comb. $\mathrm{n}$. (which has the armature of IDL setae similar to genus Coronatella). A similar trend was observed in species of Anthalona whose evolution of IDL setae are related to feeding strategies (Van Damme et al. 2011a). Thus, distinct evolutionary pressure on the food handling should be considered to explain differences observed on the IDL setae of Magnospina gen. n. species.

The morphology of the postabdomen is the most evident trait of Magnospina gen. n. in contrast to Leberis, Coronatella, Anthalona, Karualona, Extremalona, Bergamina, 
Celsinotum, Ovalona or Alona senso stricto; however, this morphological feature does not show a clear relationship with habitat and/or evolutionary history. Generally, specialized species have their morphology linked to habitat conditions (Van Damme et al. 2003, Kotov 2000a, b, Kotov 2006, Van Damme et al. 2009, Kotov et al. 2010, Van Damme and Sinev 2011, Van Damme et al. 2011a, Sinev 2014), but, apparently, this is not case of the two species from the dentifera-group, because they may occur in different kinds of habitats (Sinev and Sanoamuang 2007, Güntzel et al. 2010, Sousa et al. 2012, Kotov et al. 2013, Van Damme and Sinev 2013).

Some studies observed that the male's morphology is very important in making any inference about the relationship between closer species or between species groups in Aloninae (Sinev 1999a, 2013) as well as other cladoceran groups (Goulden 1968; Belyaeva and Taylor 2009; Kotov et al. 2009). Indeed, the morphology of the M. dentifera comb. n. male indicates more affinities with Leberis than with any genus from the Coronatella-branch. For instance, the general shape of postabdomen and antennules is similar to that described for adult males of $L$. davidi (Richard, 1895) (Sinev et al. 2005) and L. colombiensis Kotov \& Fuentes-Reines 2015 (Kotov and Fuentes-Reines 2015). However, there are clear differences between Leberis and Magnospina gen. n.: the presence of denticles on posteroventral corner of valves, shape of postabdominal claw, length of the basal spine, and armature of IDL setae (for Magnospina dentifera comb. n., Figures 30-32).

When evaluating the morphology of species of the elegans-group from Palearctic zone, Sinev et al. (2009) highlighted the morphological traits that support that this group in Coronatella-branch, as well as its presumed genus-level. Thus, the main difference between females from Magnospina gen. $\mathrm{n}$. and species of the elegans-group are related to the external morphology (shape of the body, rostrum, postabdomen and presence of denticles on the posteroventral corner of the carapace in Magnospina gen. n.); differences on the limbs are observed in the armature of IDL setae and the length of the seta 3 on the exopod of limb III. Males from the elegans-group share with Magnospina gen. $\mathrm{n}$. the presence of two lateral aesthetascs on the antennules (Figure 28), which is considered the main synapomorphy of clade Ovalonalelegans-group/Leberis (Sinev 2015a; Neretina and Sinev 2016). This confirms the phylogenetic position of Magnospina gen. n., which is closely related to Leberis.

The male of Ovalona genus also has two lateral aesthetascs on the antennules, but differs from Magnospina gen. $n$. because it has straight dorsal and ventral postabdominal margins, gonopores opening above projection to insertion of postabdominal claw, and setae 2-3 of IDL armed with setules. According to Sinev (2015a) and Neretina and Sinev (2016), Celsinotum is closer to Leberis and Ovalona, and thus, its close relationship with Magnospina gen. n. could be inferred. Celsinotum females differ quantitatively from Magnospina gen. n. in external and limb structures (see Frey 1991, Sinev and Elmoor-Loureiro 2010, Sinev and Kotov 2012). The males of Celsinotum differ from Magnospina gen. $\mathrm{n}$. in the shape of postabdomen, length of the basal spines on the postabdominal claw, presence of two-six lateral aesthetascs on antennules and setae 2-3 of IDL armed with setules (see Frey 1991, Sinev and Kotov 2012). 
Differently from Magnospina gen. n., males of the Coronatella genus have dorsal and ventral margins of the postabomen almost straight and lateral aesthetascs on antennules absent (Van Damme and Dumont 2008a, Sousa et al. 2015a). Anthalona males have a short basal spine, well-developed setules of the lateral fascicles on the postabdomen and lateral aesthetascs on antennules absent (Van Damme et al. 2011a, Sinev and Kotov 2012). Karualona males has postabdomen very similar to the one observed in Anthalona, with well-developed lateral fascicles on the postabdomen and a very short basal spine on the postabdominal claw. However, antennules of Karualona males bear one lateral and ten apical aesthetascs (see Alonso and Petrus 1989). Besides short postabdomen, the male of Extremalona has six lateral aesthetascs on antennules and well developed seta 1 of IDL and setae 2-3 armed with setules (Sinev and Shiel 2012). Differences between Magnospina gen. n. male and Bergamina cannot be stated because the male is not known, so far.

\section{Notes on Alona broaensis Matsumura-Tundisi \& Smirnov, 1984}

Alona broaensis species was described from Broa Reservoir, São Paulo, Brazil (Matsumura-Tundisi and Smirnov 1984) and it has not often been found in fauna studies conducted in many regions (including type region). The absence of some information on the morphology, including details from trunk limbs, led Van Damme et al. (2010) to list this species as a junior synonym of $M$. dentifera comb. n.. Indeed, the morphological variation observed between $M$. dentifera comb. n. populations studied here and by Rey and Vasquez (1986) for number of denticles on the posteroventral corner of carapace (Figure 8), morphology of the postabdominal claw, basal spine, and rostrum, include the features signed as diagnostic for $A$. broaensis (see Matsumura-Tundisi and Smirnov 1984). They are like those observed in description of $A$. broaensis (see Matsumura-Tundisi and Smirnov 1984). We analyzed one population identified as Alona broaensis from the Pantanal, Brazil, and morphological traits distinct from $M$. dentifera comb. n. were not observed. In the other words, there are not morphological traits that support the validity of Alona broaensis. We agree with the suggestion of Van Damme et al. (2010), and Alona broaensis is here considered as a junior synonym of $M$. dentifera comb. n..

\section{Conclusions}

Magnospina gen. n. is one more genus derived from Alona sensu lato and belongs to the Coronatella-branch, being close to Leberis, as suggested by the phylogenetic analysis based on molecular data. The synapomorphies of the Magnospina gen. n. are: (1) basal spines longer than $2 / 3$ of postabdominal claw, (2) presence of 1-4 large denticles, broad at their bases, protruding downwards, without setules between them. Magnospina gen. $\mathrm{n}$. also has a wide rostrum, prominent preanal angle at postabdomen, setae 2-3 of IDL armed with basal denticles, six setae on limb III and limb VI absent. In addition to the 
female morphology presenting consistent differences when compared to other genera from the Coronatella-branch, the male features also support the creation of this new genus that includes $M$. dentifera comb. n. and $M$. siamensis comb. $\mathrm{n}$.

\section{Acknowledgements}

We thank Prof. Dra. Adriana M. Güntzel (FUNDECT/MS), Dr. Jose Roberto Debastiani Junior and Biologist Lediane Pereira Diniz for providing the material of Alona broaensis from Pantanal and of Alona dentifera from Argentine and Pernambuco, respectively. We also thank Dr. Marcelo M. Dalosto for revising the English version of this manuscript. We are grateful to Dr. Eliana A. Panarelli, Dr. Gilmar Perbiche Neves and Dr. Ricardo Lourenço Pinto for the suggestions on the initial draft of this manuscript. We are deeply grateful to Dr. Alexey A. Kotov and Dr. Artem Yu. Sinev for the valuable criticisms, comments and suggestions that greatly improved this manuscript. The first author of this paper was awarded a grant from the Coordenação de Aperfeiçoamento de Pessoal de Nível Superior (CAPES).

\section{References}

Alonso MJ, Pretus L (1989) Alona iberica, New species: First Evidence of Noncosmopolitanism within the A. karua complex (Cladocera: Chydoridae). Journal of Crustacean Biology 9: 453-476. doi: 10.2307/1548571

Belyaeva M, Taylor DJ (2009) Cryptic species within the Chydorus sphaericus species complex (Crustacea: Cladocera) revealed by molecular markers and sexual stage morphology. Molecular Phylogenetics and Evolution 50: 534-546. doi: 10.1016/j.ympev.2008.11.007

Chatterjee T, Kotov AA, Van Damme K, Chandrasekhar SVA, Padhye S (2013) An annotated checklist of the Cladocera (Crustacea: Branchiopoda) from India. Zootaxa 3667(1): 1-89. doi: 10.11646/zootaxa.3667.1.1

Debastiani-Júnior JR, Elmoor-Loureiro LMA, Nogueira MG (2015) High taxonomic resolution as a determinant on finding new species and new record in the Río de La Plata basin: a case on Chydoridae (Crustacea: Branchiopoda: Cladocera). Nauplius 23(1): 21-30. doi: 10.1590/S0104-64972015002301

Elías-Guttiérez M, Martínez-Jerónimo F, Ivanova NV, Valdez-Moreno M, Hebert PDN (2008) DNA barcodes for Cladocera and Copepoda from Mexico and Guatemala, highlights and new discoveries. Zootaxa 1839: 1-42.

Elmoor-Loureiro LMA (2004) Phylogenetic relationships among families of the order Anomopoda (Crustacea, Branchiopoda, Cladocera). Zootaxa 760: 1-26.

Elmoor-Loureiro LMA, Santos-Wisniewski MJ, Rocha O (2013) Redescription of Alonella lineolata Sars, 1901 (Crustacea, Cladocera, Chydoridae) and its translocation to the subfamily Aloninae and to the new genus Bergamina gen. nov. Zootaxa 3630(3): 571-581. doi: 10.11646/zootaxa.3630.3.11 
Frey DG (1987) The taxonomy and biogeography of the Cladocera. Hydrobiologia 145: 5-17. doi: 10.1007/BF02530260

Frey DG (1991) A new genus of alanine chydorid cladocerans from athalassic saline waters of New South Wales, Australia. Hydrobiologia 224: 11-48. doi: 10.1007/BF00006361

Goulden CE (1968) The systematics and evolution of the Moinidae. Transactions of the American Philosophical Society Held at Philadelphia, New Series 58(6): 1-101. doi: $10.2307 / 1006102$

Güntzel AM, Panarelli EA, Silva WM, Rochhe KF (2010) Influence of connectivity on Cladocera diversity in oxbow lakes in the Taquari River floodplain (MS, Brazil). Acta Limnolgica Brasiliensia 22(1): 93-101. doi: 10.4322/actalb.02201012

Korovchinsky NM (2013) Cladocera (Crustacea: Branchiopoda) of South East Asia: history of exploration, taxon richness and notes on the zoogeography. Journal of Limnology 72(s2): 109-124. doi: 10.4081/jlimnol.2013.s2.e7

Kotov AA (2000a) Analysis of Kozhowia Vasiljeva \& Smirnov, 1969 (Chydoridae, Anomopoda, Branchiopoda), and a description of Parakozhowia n. gen. Hydrobiologia 437: 17-56. doi: 10.1023/A:1026507529975

Kotov AA (2000b) Redescription and assignment of the chydorid Indialona ganapati Petkovski, 1966 (Branchiopoda: Anomopoda: Aloninae) to Indialonini, new tribus. Hydrobiologia 439: 161-178. doi: 10.1023/A:1004187007890

Kotov AA (2006) Adaptations of Anomopoda Crustaceans (Cladocera) to the benthic mode of Life. Zoologichesky Zhurnal 85: 1043-1059. doi: 10.1134/s0013873806110157

Kotov AA, Fuentes-Reines JM (2015) A new species of Leberis Smirnov, 1989 (Cladocera: Chydoridae) from Colombia. Zootaxa 3957(5): 553-566.

Kotov AA, Ishida S, Taylor DJ (2009) Revision of the genus Bosmina Baird, 1845 (Cladocera: Bosminidae), based on evidence from male morphological characters and molecular phylogenies. Zoological Journal of the Linnean Society 156: 1-56. doi: 10.1111/j.10963642.2008.00475.x

Kotov AA, Sinev AY, Berrios VL (2010) The Cladocera (Crustacea: Branchiopoda) of six high altitude water bodies in the North Chilean Andes, with discussion of Andean endemism. Zootaxa 2430: 1-66.

Kotov AA, Taylor DJ (2011) Mesozoic fossils (>145 Mya) suggest the antiquity of the subgenera of Daphnia and their coevolution with chaoborid predators. BMC Evolutionary Biology 11: 129. doi: 10.1186/1471-2148-11-129

Kotov AA, Van Damme K, Bekker EI, Siboualipha S, Silva-Briano M, Ortiz AA, De la Rosa RG, Sanoamuang L (2013) Cladocera (Crustacea: Branchiopoda) of Vientiane province and municipality, Laos. Journal of Limnology 72(s2): 81-108. doi: 10.4081/jlimnol.2013. s2.e6

Matsumura-Tundisi T, Smirnov NN (1984) Description of Alona broaensis sp. n. (Crustacea: Cladocera). Hydrobiologia 113: 327-329. doi: 10.1007/BF00026619

Neretina AN, Sinev AY (2016) A revision of the genus Leberis Smirnov, 1989 (Cladocera: Chydoridae) in the Old World and Australia. Zootaxa 4079: 501-533. doi: 10.11646/ zootaxa.4079.5.1 
Rajapksa R, Fernando CH (1987) A redescription of Euryalona orientalis (Daday, 1898), with a consideration of the other species in the genus Euryalona (Cladocera: Chydoridae). Hydrobiologia 150: 75-90. doi: 10.1007/BF00006611

Rey J, Vasquez E (1986) Cladocères de quelques corps d'eaux du basin moyen de l'Orénoque (Vénézuéla) Annales de Limnologie - International Journal of Limnology 22(2): 137-168. doi: $10.1051 /$ limn/1986013

Sacherová V, Hebert PDN (2003) The evolutionary history of the Chydoridae (Crustacea: Cladocera). Biological Journal of the Linnean Society 79: 629-643. doi: 10.1046/j.10958312.2003.00216.x

Sinev AY (1999a) Alona werestchangini sp.n., a new spcies of the genus Alona Baird, 1843 related to A. guttata Sars, 1862 (Anomopoda: Chydoridae). Arthropoda Selecta 8: 23-30.

Sinev AY (1999b) Alona costata Sars, 1862 versus related palaeotropical species: the first example of close relations between species with a different number of main head pores among Chydoridae (Crustacea: Anomopoda). Arthropoda Selecta 8: 131-148.

Sinev AY (2001a) Redescription of Alona iheringi Sars, 1901 (Chydoridae, Anomopoda, Branchiopoda), a South American species related to A. rustica Scott, 1895. Hydrobiologia 464: 113-119. doi: 10.1023/A:1013935100483

Sinev AY (2001b) Redescription of Alona glabra Sars, 1901, a South American species of the pulchella-group (Chydoridae, Anomopoda, Branchiopoda). Arthropoda Selecta 10: 273-280.

Sinev AY (2001c) Separation of Alona cambouei Guerne \& Richard, 1893 from Alona pulchella King, 1853 (Branchiopoda, Anomopoda, Chydoridae). Arthropoda Selecta 10: 5-18.

Sinev AY (2008) A new species related to Alona costata Sars, 1862 (Cladocera: Anomopoda: Chydoridae) from South Africa. Zootaxa 1707: 23-36.

Sinev AY (2009) Notes on morphology and taxonomic status of some North American species of the genus Alona Baird, 1843 (Cladocera: Anomopoda: Chydoridae). Fundamental Applied Limnology 175: 59-77. doi: 10.1127/1863-9135/2009/0175-0059

Sinev AY (2013) Cladocerans of Alona affinis group (Cladocera: Anomopoda: Chydoridae) from North America. Zootaxa 3693: 329-343. doi: 10.11646/zootaxa.3693.3.3

Sinev AY (2014) A comparative morphological analyses of four species of Camptocercus Baird, 1843 (Cladocera: Anomopoda: Chydoridae). Zootaxa 3895: 183-207. doi: 10.11646/ zootaxa.3895.2.3

Sinev AY (2015a) Revision of the puchella-group of Alona s. lato leads to its translocation to Ovalona Van Damme et Dumont, 2008 (Branchiopoda: Anomopoda: Chydoridae). Zootaxa 4044: 451-492. doi: 10.11646/zootaxa.4044.4.1

Sinev AY (2015b) Morphology and phylogenetic position of three species of genus Camptocercus Baird, 1843 (Cladocera: Anomopoda: Chydoridae). Zootaxa 4040: 169-186. doi: 10.11646/zootaxa.4040.2.4

Sinev AY, Elmoor-Loureiro LMA (2010) Three new species of chydrorid cladocerans of subfamily Aloninae (Brachiopoda: Anomopoda: Chydoridae) from Brazil. Zootaxa 2390: $1-25$.

Sinev AY, Kotov AA (2012) New and rare Aloninae (Cladocera: Anomopoda: Chydoridae) from Indochina. Zootaxa 3334: 1-28. 
Sinev AY, Sanoamuang L (2007) Alona siamensis sp. n., a new species of Cladocera from SouthEast Asia, related to Alona dentifera (Sars, 1901) (Anomopoda: Chydoridae). Arthropoda Selecta 16: 143-150.

Sinev AY, Shiel RJ (2012) Extremalona timmsi gen. nov., sp. nov., a new cladoceran (Cladocera: Anomopoda: Chydoridae) from an acid saline lake in southwest Western Australia. Journal of Natural History 46: 45-46. doi: 10.1080/00222933.2012.727215

Sinev AY, Silva-Briano M (2012) Cladocerans of genus Alona Baird, 1843 (Cladocera: Anomopoda: Chydoridae) and related genera from Aguascalientes State, Mexico. Zootaxa 3569: 1-24.

Sinev AY, Alonso M, Sheveleva LG (2009) New species of Alona from South-East Russia and Mongolia related to Alona salina Alonso, 1996 (Cladocera: Anomopoda: Chydoridae). Zootaxa 2326: 1-23.

Sinev AY, Kotov AA, Van Damme K (2004) Morphology of a Neotropical cladoceran Alona dentifera (Sars, 1901), and its position within the Chydoridae Stebbing, 1902 (Branchiopoda: Anomoda). Arthropoda Selecta 13: 99-107.

Sinev AY, Van Damme K, Kotov AA (2005) Redescription of tropical-temperate cladocerans Alona diaphana King, 1853 and Alona davidi Richard, 1895 and their translocation to Leberis Smirnov, 1989 (Branchiopoda: Anomopoda: Chydoridae). Arthropoda Selecta 14(3): 183-205.

Sousa FDR, Elmoor-Loureiro LMA (2012) How many species of cladocerans (Crustacea, Branchiopoda) are found in Brazilian Federal District? Acta Limnologica Brasiliensia 24(4): 351-362. doi: 10.1590/S2179-975X2013005000008

Sousa FDR, Elmoor-Loureiro LMA, Santos S (2015a) Redescription of Coronatella poppei (Richard, 1897) (Crustacea, Branchiopoda, Chydoridae) and a revision of the genus in Brasil, with description of new taxa. Zootaxa 3955(2): 211-244. doi: 10.11646/ zootaxa.3955.2.3

Sousa FDR, Elmoor-Loureiro LMA, Santos S (2015b) Alona kaingang sp. nov (Crustacea, Cladocera, Chydoridae): a new species of the pulchella-group, with identification key to Neotropical species. Zoological Studies 54(48): 1-12.

Van Damme K, Dumont HJ (2008a) Further division of Alona Baird, 1843: separation and position of Coronatella Dybowski \& Grochowski and Ovalona gen.n. (Crustacea: Cladocera). Zootaxa 1960: 1-44.

Van Damme K, Dumont HJ (2008b) The 'true' genus Alona Baird, 1843 (Crustacea: Cladocera: Anomopoda): characters of the A. quadrangularis-group and description of a new species from Democratic Republic Congo. Zootaxa 1945: 1-25.

Van Damme K, Eggermont H (2011) The Afromontane Cladocera (Crustacea: Branchiopoda) of the Rwenzori (Uganda-D. R. Congo): taxonomy, ecology and biogeography. Hydrobiologia 675: 57-100. doi: 10.1007/s10750-011-0892-0

Van Damme K, Sinev AY (2011) A new genus of cave-dwelling microcrustaceans from the Dinaric Region (south-east Europe): adaptations of true stygobitic Cladocera (Crustacea: Branchiopoda). Zoological Journal of Linnenan Society 161: 31-52. doi: 10.1111/j.10963642.2010.00639.x 
Van Damme K, Sinev AY (2013) Tropical Amphi-Pacific disjunctions in Cladocera (Crustacea: Branchiopoda). Journal of Limnology 72 (s2): 209-244. doi: 10.4081/jlimnol.2013.s2.e11

Van Damme K, Bekker E, Kotov AA (2013) Endemism in the Cladocera (Crustacea: Branchiopoda) of Southern Africa. Journal of Limnology 72: 440-463. doi: 10.4081/jlimnol.2013.e36

Van Damme K, Brancelj A, Dumont HJ (2009) Adaptations to the hyporheic in Aloninae (Crustacea: Cladocera): allocation of Alona protzi Hartwig, 1900 and related species to Phreatalona gen. nov. Hydrobiologia 618: 1-34. doi: 10.1007/s10750-008-9607-6

Van Damme K, Elías-Gutiérrez M, Dumont HJ (2011b) Three rare European “Alona” taxa (Branchiopoda: Cladocera: Chydoridae), with notes on distribution and taxonomy. Annales de Limnologie - International Journal of Limnology 47: 45-63. doi: 10.1051/ $\operatorname{limn} / 2010034$

Van Damme K, Kotov AA, Dumont HJ (2010) A checklist of names in Alona Baird 1843 (Crustacea: Cladocera: Chydoridae) and their current status: an analysis of the taxonomy of a lump genus. Zootaxa 2330: 1-63.

Van Damme K, Sinev AY, Dumont HJ (2011a) Separation of Anthalona gen.n. from Alona Baird, 1843 (Branchiopoda: Cladocera: Anomopoda): morphology and evolution of scraping stenothermic Alonine. Zootaxa 2875: 1-64.

Van Damme K, Chiambeng G, Maiphae S, Dumont HJ (2003) New species in the rheophilous genus Nicsmirnovius Chiambeng\&Dumont, 1999 (Branchiopoda: Anomopoda: Chydoridae) and reassignment of Alona eximia Kiser, 1948 and Alonella fitzpatricki Chien, 1970. Hydrobiologia 499: 25-49. doi: 10.1023/A:1026391501312 